\title{
Staging of pulmonary metastases using dual-energy computed tomography after anti-angiogenic therapy
}

\author{
Jens-Christian Altenbernd ${ }^{* 1}$, Axel Wetter ${ }^{2}$, Lale Umutlu², Michael Forsting ${ }^{2}$ \\ ${ }^{1}$ Knappschaftskrankenhaus Recklinghausen, Recklinghausen, Germany \\ ${ }^{2}$ University Hospital Essen, Essen, Germany
}

Received: May 4, 2017

DOI: $10.5430 /$ ijdi.v4n2p74
Accepted: July 19, 2017

URL: https://doi.org/10.5430/ijdi.v4n2p74

\begin{abstract}
Objective: The aim of this study was the evaluation of dual-energy computed tomography (DECT) for the assessment of pulmonary metastases (PM) after antiangiogentic therapy (AT).

Material and methodology: A total of 82 patients with non-small cell lung carcinoma (NSCLC), colorectal cancer (CRC), gastrointestinal stromal tumors (GIST) and hepatocellular carcinoma (HCC) were examined before and after AT with DECT of the lung. The number, size, CT densities (HU) of the PM were determined by 2 radiologists in consens in both DECT. The Wilcoxon sign rank test was applied (SPSS, version 21, SPSS, IBM, Chicago, USA).

Results: The 82 patients (NSCLC: 32/82; CRC: 34/82; GIST: 10/82; HCC: 6/82) with a total of 201 PM were included. DECT were produced with a time interval of $4 \pm 1$ months. Size changes of the metastases: PM total $23 \mathrm{~mm}$ vs. $24 \mathrm{~mm} ; p=.1 / \mathrm{NSCLC}$ $22 \mathrm{~mm}$ vs. $23 \mathrm{~mm} ; p=.2 /$ CRC $23 \mathrm{~mm}$ vs. $23 \mathrm{~mm} ; p=.3 /$ GIST $24 \mathrm{~mm}$ vs. $25 \mathrm{~mm} ; p=.1 /$ HCC $22 \mathrm{~mm}$ vs. $21 \mathrm{~mm} ; p=.1$. Contrast media in the course: PM total $45 \mathrm{HU}$ vs. $25 \mathrm{HU} ;-44 \% ; p<.05 /$ NSCLC $43 \mathrm{HU}$ vs. $22 \mathrm{HU} ;-49 \% ; p<.05 / \mathrm{CRC} 33 \mathrm{HU}$ vs. $15 \mathrm{HU} ;-55 \% ; p<.05 /$ GIST $45 \mathrm{HU}$ vs. $24 \mathrm{HU} ; 47 \% ; p<.05 / \mathrm{HCC} 62 \mathrm{HU}$ vs. $43 \mathrm{HU} ;-31 \%, p<.05$.

Conclusions: The quantification of the contrast medium uptake of pulmonary metastases is valid by using dual-energy imaging. In this way, the therapy response according to antiangiogenetic therapy with regard to the contrast medium uptake can be assessed more precisely without native imaging in addition to changes in the size of the metastases.
\end{abstract}

Key Words: Dual-energy computed tomography, Pulmonary metastases, Antiangiogentic therapy

\section{INTRODUCTION}

The response of malignant tumors to therapy is usually investigated by means of computed tomography or magnetic resonance imaging. To be documented here are not only changes in size, but also changes in tumor perfusion. A reduction of tumor perfusion given a constant or even increasing size can sometimes be assessed as response to therapy if "pseudo progress" is evident, for example, in view of tumour necrosis brought about during therapy. Essential here therefore is a quantification of the tumors' uptake of contrast medium, which can be calculated and visualized by means of dual-energy CT (DECT). ${ }^{[1]}$

For years, DECT has been an established diagnostic procedure with a number of advantages over mono-energy computed tomography. Tissue differentiation can be carried out successfully. Virtual subtraction of calcium or contrast medium with resultant reduction in radiation exposure and accurate quantification including visualization of contrast

\footnotetext{
${ }^{*}$ Correspondence: Jens-Christian Altenbernd; Email: jens.altenbernd@gmail.com; Address: Knappschaftskrankenhaus Recklinghausen, Recklinghausen, Germany.
} 
medium uptake. ${ }^{[2-6]}$

Already in 1971, Folkmann et al. dealt with tumor angiogenesis in terms of therapeutic treatment options. ${ }^{[7]}$ However, bevacizumab was approved only in $2004 .^{[8]}$

In the following years, an increasing number of antiangiogenic therapies established themselves for cases such as those involving NSCLC, HCC, GIST and CRC. ${ }^{[9-12]}$

The aim of this study was to evaluate dual-energy CT for assessing pulmonary metastases of malignant tumors with respect to contrast medium uptake as part of anti-angiogenic therapy.

\section{MATERIAls AND METHODOLOGY}

Between 4/2012 and 10/2013, 82 patients (46 women, 36 men, average age $63 \pm 12$ years) with pulmonary metastases were examined consecutively. Included as primary tumours were non-small cell lung carcinoma (NSCLC), colorectal cancer (CRC), gastrointestinal stromal tumors (GIST) and hepatocellular carcinoma (HCC). The primary tumors were all surgically removed before the 1 st DECT $(13 \pm 5$ months); the pulmonary metastases involved new secondary growths in the course of time. There were no further extrapulmonary secondary growths. The inclusion criterion was chemotherapy conducted between the 1st and 2nd DECT using anti-VEGF MTTs (vascular endothelial growth factor - molecular targeted therapies). The dual-energy CT tests were carried out as part of the standardized staging checks. 2 DECTs were performed for every patient. The first DECT was always performed before start of chemotherapy, the next examination being conducted after chemotherapy.

The number and size of the secondary growths were determined by consensus. So were the secondary growths' densities with the help of dual-energy software. A differentiation was made here between virtual native density values and absolute density values after administration of the contrast medium.

The study had been previously approved by the ethics commission and all patients had given their consent. The CT examination results were acquired using a dual-source multidetector CT (Somatom Definition Dual Source; Siemens medical solutions, Forchheim, Germany). Non-ionic contrast medium was injected in the supine position $(1.5 \mathrm{ml}$ per kilogram of body weight, Xenetix 300, Guerbet, Sulzbach, Germany).

The data records were obtained in the arterial phase using bolus tracking from the diaphragm to the top of the lungs during inspiration. Examination parameters of tubes A/B: $140 \mathrm{kV} / 80 \mathrm{kV}$ and $96 \mathrm{mAs} / 404 \mathrm{mAs}$.

\subsection{Post-processing and image data reconstruction}

The DECT generated 3 image data sets: $80 \mathrm{kV}, 140 \mathrm{kV}$ data sets, hybrid images incorporating $60 \%$ of the $140 \mathrm{kV}$ data and $40 \%$ of the $80 \mathrm{kV}$ data.

For post-processing, the image data were loaded on to a workstation (syngo MMWP; Siemens Medical Solutions, Forchheim, Germany). At this workstation, a special postprocessing software was used to generate image data sets with a special representation of contrast medium uptake (overlay image data).

\subsection{CT measurement of pulmonary nodule density}

The overlay image data of the 1st and 2nd DECTs were compared by two radiologists (13/15 years of experience), the results being in consensus: CT density values (HU) for pulmonary nodules (min. $11 \mathrm{~mm}$, max. $35 \mathrm{~mm}$ diameter, maximum size within the pulmonary nodule).

For the comparison between the CT density values of the virtual native and overlay DECT image data, the Wilcoxon signed rank test was employed, and the results checked for statistical relevance. All calculations were performed using a statistical software (SPSS, version 21; SPSS, IBM, Chicago, USA). A $p$-value of $<.05$ exhibited a statistical significance.

The tumors was assessed as pulmonary metastases after a comparison with mono-energy computed tomographies prior to the study (55/82) or through histological verification $(27 / 82)$.

\section{Results}

From 4/2012 to $10 / 2013,82$ patients with a total of 201 pulmonary metastases $(2.4 \pm 0.9$ metastases per patient) were examined consecutively. Primary tumors were NSCLC (32/82), CRC (34/82), GIST(10/82) and HCC (6/82). The dual-energy CT tests were carried out as part of the standardized staging checks. 2 DECT scans were performed at a chronological interval of $4 \pm 1$ months for every patient. The first DECT was always performed before start of chemotherapy, the next examination being conducted after chemotherapy. Histological verifications of the metastases were carried out for 27 of the 82 patients. The remaining tumors (55/82) were assessed as pulmonary metastases after comparison with mono-energy computed tomographies before the 1st DECT ( $5 \pm 2$ months).

The changes in the metastases' size in the course of time are listed in Table 1. Evident both in terms of total number (23 $\mathrm{mm}[11-35 \mathrm{~mm}]$ vs. $19 \mathrm{~mm}[7-31 \mathrm{~mm}] ;-17 \% ; p=.1)$ as well as metastases in dependence on the primary tumor was a regressive average metastasis diameter in each case, but without any statistical significance (NSCLC $22 \mathrm{~mm}$ [12-34 
$\mathrm{mm}]$ vs. $19 \mathrm{~mm}$ [8-30 mm]; -14\%; $p=.15 / \mathrm{CRC} 23 \mathrm{~mm}$ [11-35 mm] vs. $19 \mathrm{~mm}[8-30 \mathrm{~mm}] ;-17 \% ; p=.3 /$ GIST 24 $\mathrm{mm}[13-30 \mathrm{~mm}]$ vs. $22 \mathrm{~mm}[8-29 \mathrm{~mm}] ;-8 \% ; p=.1 / \mathrm{HCC}$ $22 \mathrm{~mm}$ [13-34 mm] vs. $18 \mathrm{~mm}$ [8-30 mm]; -18\%; $p=.09)$.

The contrast-medium uptakes of the metastases are represented in Table 2. Evident in the contrast-medium uptakes of the metastases are significant regressions both in terms of total number $(45 \mathrm{HU}( \pm 4)$ vs. $25 \mathrm{HU}( \pm 5) ;-44 \% ; p<.05)$ and with respect to the primary tumors (NSCLC $43 \mathrm{HU}( \pm 5)$ vs. $22 \mathrm{HU}( \pm 6) ;-49 \% ; p<.05 / \mathrm{CRC} 33 \mathrm{HU}( \pm 5)$ vs. 15 HU ( \pm 4$) ;-55 \% ; p<.05 /$ GIST 45 HU $( \pm 4)$ vs. $24 \mathrm{HU}( \pm 5)$; $-47 \% ; p<.05 / \mathrm{HCC} 62 \mathrm{HU}( \pm 4)$ vs. $43 \mathrm{HU}( \pm 5) ;-31 \%, p<$ $.05)$.

Table 1. Average size $(\mathrm{mm})$ of the pulmonary secondary growths in total and in dependence on the primary tumor in the course of time, determined using dual-energy CT

\begin{tabular}{|c|c|c|c|c|}
\hline & Quantity & $\begin{array}{l}\text { Average size in } \mathrm{mm} \\
\text { (min.-max. diameter) } \\
\text { Baseline }\end{array}$ & $\begin{array}{l}\text { Average size in mm } \\
\text { (min.-max. diameter) } \\
\text { Follow up }\end{array}$ & $p$ \\
\hline Total secondary growths & 201 & $23(11-35)$ & $19(7-31)$ & .1 \\
\hline Secondary growths in the case of NSCLC & 75 & $22(12-34)$ & $19(8-30)$ & .15 \\
\hline Secondary growths in the case of CRC & 84 & $23(11-35)$ & $20(7-31)$ & .3 \\
\hline Secondary growths in the case of GIST & 26 & $24(13-30)$ & $22(8-29)$ & .1 \\
\hline Secondary growths in the case of HCC & 16 & $22(13-34)$ & $18(8-30)$ & .09 \\
\hline
\end{tabular}

Table 2. Average contrast-medium uptake (HU) of the pulmonary secondary growths in total and in dependence on the primary tumor in the course of time, determined using dual-energy CT

\begin{tabular}{|c|c|c|c|c|}
\hline & Quantity & $\begin{array}{l}\text { Average contrast-medium } \\
\text { uptake in HU (+/- standard } \\
\text { deviation) } \\
\text { Baseline DECT }\end{array}$ & $\begin{array}{l}\text { Average contrast-medium } \\
\text { uptake in HU (+/- standard } \\
\text { deviation) } \\
\text { Follow up DECT }\end{array}$ & $p$ \\
\hline Total secondary growths & 201 & $45(4)$ & $25(5)$ & $<.05$ \\
\hline Secondary growths in the case of NSCLC & 75 & $43(5)$ & $22(6)$ & $<.05$ \\
\hline Secondary growths in the case of CRC & 84 & $33(6)$ & $15(4)$ & $<.05$ \\
\hline Secondary growths in the case of GIST & 26 & $45(4)$ & $24(5)$ & $<.05$ \\
\hline Secondary growths in the case of HCC & 16 & $62(4)$ & $43(5)$ & $<.05$ \\
\hline
\end{tabular}

\section{DISCUSSION AND CONCLUSION}

An assessment of the chronology of pulmonary metastases, taking into consideration modified tumor perfusions after anti-angiogenic therapy, poses a new challenge to radiological diagnosis. ${ }^{[1]}$ Additional assessment techniques are therefore necessitated not only by the mechanism of action, but also the criteria of therapy response. ${ }^{[13]}$

Our study was able to demonstrate that quantification of contrast-medium uptake is possible and valid using dualenergy imaging.

A very important aspect here is that previous mono-energy quantifications were only possible after acquisition of native image data, this being associated with increased radiation exposure. What we find remarkable is that perfusion of the secondary pulmonary tumors in our group of patients is significantly reduced during anti-angiogenetic therapy, regardless of the primary tumor, whereas the tumor diameters also exhibit a reduction, but without statistical significance (see Figures 1-4). This is an important additional item of information in assessing therapy response, and can prevent premature termination of a therapy which is inherently successful.

Other studies have presented modified criteria for assessing size during therapy. For example, Krajewski et al. recommend a $10 \%$ reduction in size for renal cell carcinoma during anti-angiogenic therapy as a successful therapy response, contrary to the usual RECIST criteria. ${ }^{[14]}$ Even if our results need to be checked in further studies, there is legitimate hope that the assessment of therapy response should be multimodal, that is, taking into account changes in tumor size and morphology in combination with clinical parameters. ${ }^{[3,15,16]}$

Chae et al. dealt with the characterization of singular pulmonary nodules using dual-energy CT. They conclude that this technique has a number of applications in computed 
tomography which makes use of contrast media, and that differentiation between benign and malignant lesions is more successful. ${ }^{[5,6]}$

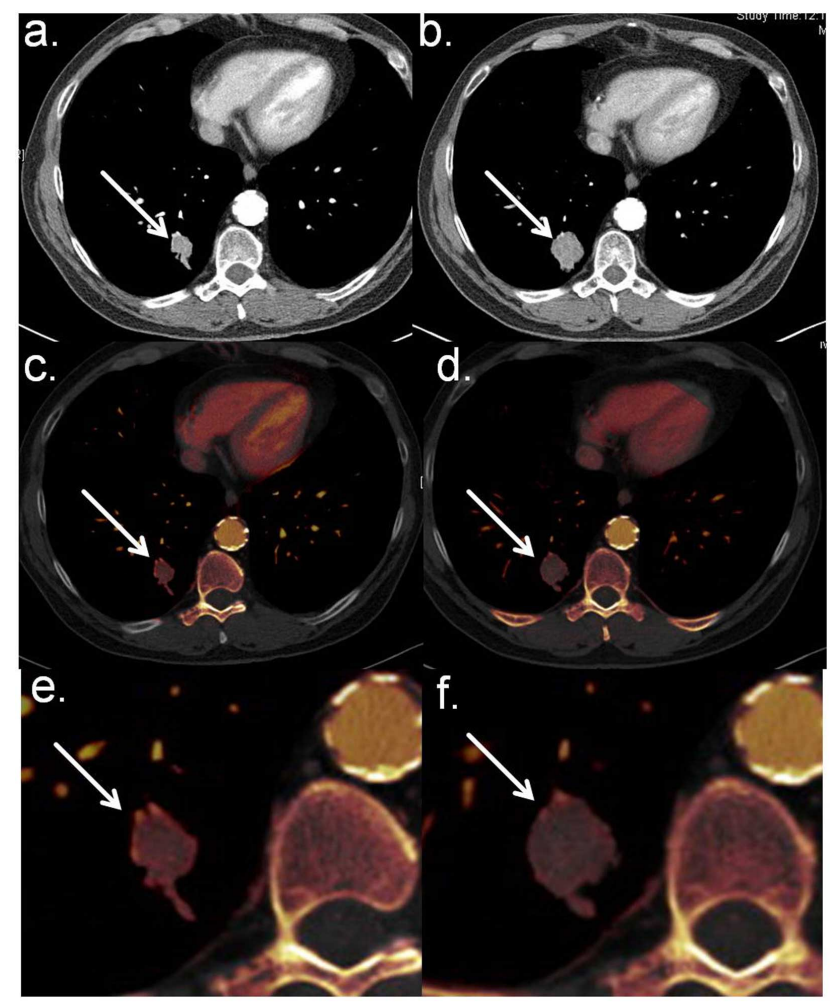

Figure 1. Pulmonary metastases examined before and after first cycle of chemotherapy using dual-energy computed tomography. Minor reduction in size and reduction of contrast-medium uptake in the course of time.

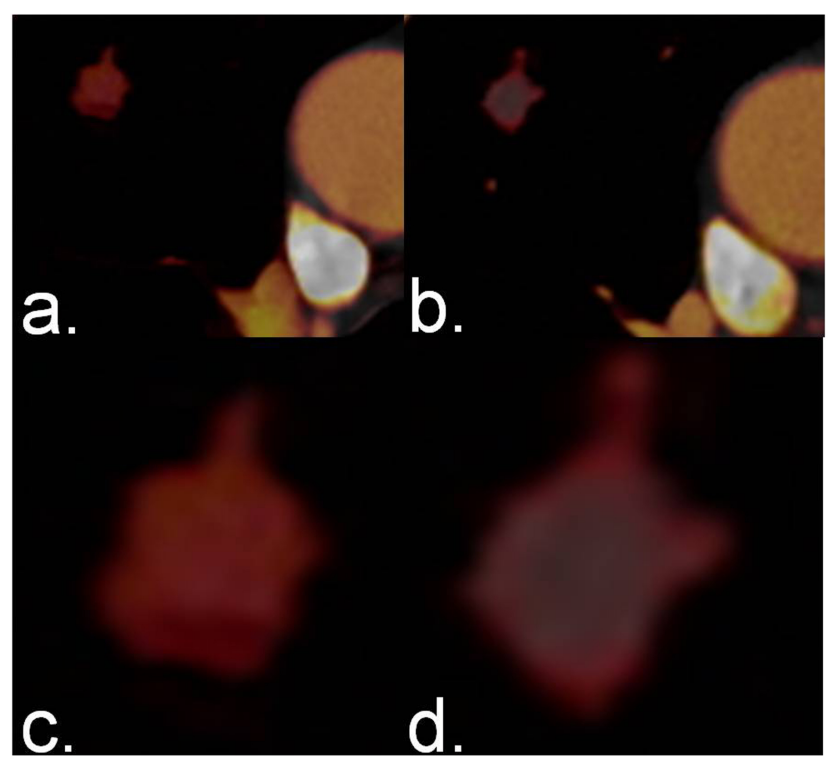

Figure 2. Secondary pulmonary growths of constant size in course of time, case involving NSCLC, with reduced uptake of contrast medium

Published by Sciedu Press

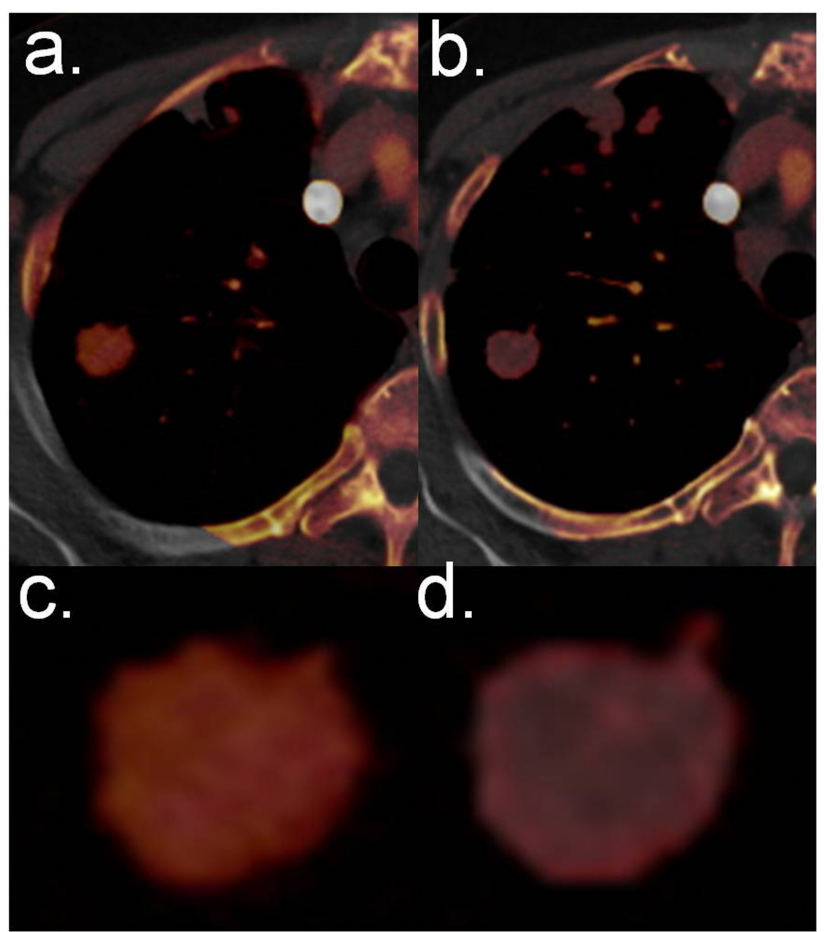

Figure 3. Secondary pulmonary growths of constant size in the course of time, case involving GIST, with reduced uptake of contrast medium

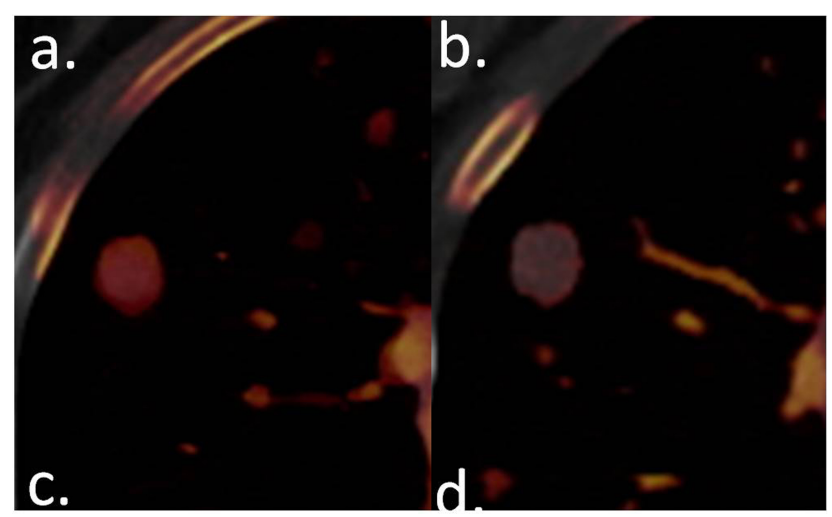

Figure 4. Secondary pulmonary growths of constant size in the course of time, case involving HCC, with reduced uptake of contrast medium

According to Ogawa et al., dual-energy CT image data created $60 \mathrm{~s}$ after injection of contrast medium provide an excellent hilar and mediastinal contrast of vessel lymph nodes and can replace two-phase scan records. ${ }^{[3]}$

With previous studies, we were able to demonstrate the significance of DECT in assessing therapy response after radioembolization of the liver, and assessing pulmonary metastases in terms of the contrast medium's dynamics. ${ }^{[17,18]}$

DECT has a wide range of potential applications in the field of oncology. ${ }^{[19,20]}$ The response to anti-angiogenic therapy 
in the case of pulmonary metastases examined by us was not studied previously, however.

In the case of gastrointestinal stromal tumors, DECT has proven a useful supplement to RECIST and $\mathrm{CHOI}^{[21,22]}$ for monitoring therapy within the framework of studies.

The response of patients with HCC to therapy using sorafenib was assessed by Dai et al. using volumetric iodine intake based on DECT data, and considered as a useful additional modality.

Another factor to be taken into account is the precise time of data acquisition after administration of contrast medium, this having been investigated by Thaiss et al. in the case of patients with HCC and lymphomas using dual-energy CT, taking into consideration the concentration of iodine. ${ }^{[2]}$
A limitation of our study is assessment of a single follow-up DECT in each case. Future studies should include longer observation periods. Another disadvantage of our study is the lack of correlation with clinical parameters as well as the overall survival of patients. Further studies should examine the extent to which changes in the tumors' uptake of contrast medium have clinical relevance, and whether they are reflected in prolonged survival. Only this would allow dependable review of therapy response criteria.

In summary, the dual-energy computed tomography is a useful instrument for assessing response to anti-angiogenetic therapy for pulmonary metastases.

\section{CONFlicts of InTEREST Disclosure}

The authors have no conflict of interest related to this publication.

\section{REFERENCES}

[1] Tirumani SH, Fairchild A, Krajewski KM, et al. Anti-VEGF molecular targeted therapies in common solid malignancies: comprehensive update for radiologists. Radiographics. 2015; 35: 455-474. PMid:25763729 https : //doi .org/10.1148/rg. 352140119

[2] Thaiss WM, Haberland U, Kaufmann S, et al. Iodine concentration as a perfusion surrogate marker in oncology: Further elucidation of the underlying mechanisms using Volume Perfusion CT with 80 kVp. Eur Radiol. 2016; 26: 2929-2936. PMid:26679179 https://doi.org/10.1007/s00330-015-4154-9

[3] Ogawa M, Hara M, Imafuji A, et al. Dual-energy CT can evaluate both hilar and mediastinal lymph nodes and lesion vascularity with a single scan at 60 seconds after contrast medium injection. Acad Radiol. 2012; 19: 1003-1010. PMid:22621917 https : //doi.org/10.1016/j.acra.2012.03.024

[4] Kang MJ, Park CM, Lee CH, et al. Dual-energy CT: clinical applications in various pulmonary diseases. Radiographics. 2010; 30: 685698. PMid:20462988 https://doi.org/10.1148/rg. 3030951 01

[5] Chae EJ, Song JW, Krauss B, et al. Dual-energy computed tomography characterization of solitary pulmonary nodules. J Thorac Imaging. 2010; 25: 301-310. PMid:21042068 https://doi .org/10.1097/ RTI.0b013e3181e16232

[6] Chae EJ, Song JW, Seo JB, et al. Clinical utility of dual-energy CT in the evaluation of solitary pulmonary nodules: initial experience. Radiology. 2008; 249: 671-681. PMid:18796658 https: //doi.org/10.1148/radiol.2492071956

[7] Folkman J. Tumor angiogenesis: therapeutic implications. N Engl J Med. 1971; 285: 1182-1186. PMid:4938153 https : //doi .org/ 10.1056/NEJM197111182852108

[8] Cohen MH, Gootenberg J, Keegan P, et al. FDA drug approval summary: bevacizumab plus FOLFOX4 as second-line treatment of colorectal cancer. Oncologist. 2007; 12: 356-361. PMid:17405901 https ://doi .org/10.1634/theoncologist. 12-3-356

[9] Gutierrez M, Giaccone G. Antiangiogenic therapy in nonsmall cell lung cancer. Curr Opin Oncol. 2008; 20: 176-182. PMid:18300767 https://doi.org/10.1097/CCO.0b013e3282f4e55e
[10] Majithia N, Grothey A. Regorafenib in the treatment of colorectal cancer. Expert Opin Pharmacother. 2016; 17: 137-145. PMid:26559195 https://doi .org/10.1517/14656566.2016.1118054

[11] Smith AD, Lieber ML, Shah SN. Assessing tumor response and detecting recurrence in metastatic renal cell carcinoma on targeted therapy: importance of size and attenuation on contrast-enhanced CT. AJR Am J Roentgenol. 2010; 194: 157-165. PMid:20028918 https://doi.org/10.2214/AJR.09.2941

[12] Yamada T, Ohtsubo K, Izumi K, et al. Metastatic renal cell carcinoma complicated with diffuse alveolar hemorrhage: a rare adverse effect of sunitinib. Int J Clin Oncol. 2010; 15: 638-641. PMid:20602135 https://doi.org/10.1007/s10147-010-0104-3

[13] Smith AD, Shah SN, Rini BI, et al. Utilizing pre-therapy clinical schema and initial CT changes to predict progression-free survival in patients with metastatic renal cell carcinoma on VEGF-targeted therapy: a preliminary analysis. Urol Oncol. 2013; 31: 1283-1291. PMid:21956044 https ://doi.org/10.1016/j.urolonc. 2011 .08 .010

[14] Krajewski KM, Franchetti Y, Nishino M, et al. 10\% Tumor diameter shrinkage on the first follow-up computed tomography predicts clinical outcome in patients with advanced renal cell carcinoma treated with angiogenesis inhibitors: a follow-up validation study. Oncologist. 2014; 19: 507-514. PMid:24755461 https : //doi.org/10.1634/theoncologist.2013-0391

[15] Jung SG, Kim JH, Kim HS, et al. Tumor response assessment by the single-lesion measurement per organ in small cell lung cancer. Chin J Cancer Res. 2016; 28: 161-167. PMid:27199513 https: //doi.org/10.21147/j.issn.1000-9604.2016.02.03

[16] van Persijn van Meerten EL, Gelderblom H, Bloem JL. RECIST revised: implications for the radiologist. A review article on the modified RECIST guideline. Eur Radiol. 2010; 20: 1456-1467. PMid:20033179 https : //doi.org/10.1007/s00330-009-168 5-y

[17] Altenbernd J, Wetter A, Forsting M, et al. Treatment response after radioembolisation in patients with hepatocellular carcinoma-An evaluation with dual energy computed-tomography. Eur J Radiol Open. 2016; 3: 230-235. PMid:27622200 https ://doi.org/10.1016/ j.ejro.2016.08.002 
[18] Altenbernd J, Wetter A, Umutlu L, et al. Dual-energy computed tomography for evaluation of pulmonary nodules with emphasis on metastatic lesions. Acta Radiol. 2016; 57: 437-443. PMid:25907120 https://doi.org/10.1177/0284185115582060

[19] Heye T, Nelson RC, Ho LM, et al. Dual-energy CT applications in the abdomen. AJR Am J Roentgenol. 2012; 199: S64-70. PMid:23097169 https://doi.org/10.2214/AJR.12.9196

[20] De Cecco CN, Darnell A, Rengo M, et al. Dual-energy CT: oncologic applications. AJR Am J Roentgenol. 2012; 199: S98-S105. PMid:23097174 https://doi.org/10.2214/AJR.12.9207
[21] Meyer M, Hohenberger P, Apfaltrer P, et al. CT-based response assessment of advanced gastrointestinal stromal tumor: dual energy CT provides a more predictive imaging biomarker of clinical benefit than RECIST or Choi criteria. Eur J Radiol. 2013; 82: 923-928. PMid:23410905 https://doi.org/10.1016/j.ejrad.2013.0 1.006

[22] Schramm N, Schlemmer M, Englhart E, et al. Dual energy CT for monitoring targeted therapies in patients with advanced gastrointestinal stromal tumor: initial results. Curr Pharm Biotechnol. 2011; 12: 547-557. PMid:21342100 https://doi.org/10.2174/1389 20111795164066 